\title{
Routine intrauterine device checks: are they advisable?
}

\author{
Isabel B Draper, ${ }^{1}$ M Sayeed Haque, ${ }^{2}$ Richard J McManus ${ }^{3}$
}

\begin{abstract}
1 General Practitioner, Whitehall Medical Practice, Rugby, UK

${ }^{2}$ Medical Statistician, Department of Primary Care Clinical Sciences, University of Birmingham, Birmingham, UK ${ }^{3}$ General Practitioner and Professor of Primary Care Research, Department of Primary Care Clinical Sciences, University of Birmingham, Birmingham, UK
\end{abstract}

\section{Correspondence to} Dr Isabel B Draper, Whitehall Medical Practice, Morton Gardens, Rugby CV21 3AQ, UK; ibd@doctors.org.uk

Received 20 May 2010 Accepted 7 March 2011 Published Online First 1 August 2011

\begin{abstract}
Background Patients using the intrauterine contraceptive device (IUD) were previously advised to undergo routine checks; in 2004, the National Institute for Health and Clinical Excellence stated the practice was unnecessary. This study was conducted to examine the evidence for this advice.

Methods A retrospective examination of case records of patients of Whitehall Medical Practice, Rugby, UK who had used an IUD for a minimum of 2 years was performed. Data were extracted concerning demographic details, types of IUD used, dates of their use and of any checks, defaults from checks and side effects. Kaplan-Meier survival analysis was performed to compare outcomes in frequent and infrequent attenders, and in frequent and infrequent defaulters from checks.

Results The study population comprised 272 individuals using a total of 423 devices. Frequent check attenders showed adverse events earlier, or at no significant time difference, to infrequent attenders.

Conclusions Considering patients who use an IUD for a minimum of 2 years, this study found no evidence of harm occurring in those who attended infrequently compared to frequent attenders. If these data from a single practice are generalisable, after an initial check following insertion, women can be asked to attend as needed and only be recalled for smears and at the end of the life of the IUD.
\end{abstract}

\section{Introduction}

Until recently, it has been usual for a patient using the intrauterine device (IUD) or intrauterine system (IUS) for contraception to be advised to have an annual check. The provision of a fee for providing this service was confirmed in the new contract for general practitioners (GPs) in 2004, and guidelines continue to recommend the practice. ${ }^{12}$ However, under the increasing pressure of workload and financial constraints, annual checks have been progressively abandoned by many

\section{Key message point}

Regular routine intrauterine device checks do not appear to confer any protection to the user from adverse events.

centres and the National Institute for Health and Clinical Excellence stated in 2004 that the practice was unnecessary. ${ }^{3}$

Evidence that annual checks were beneficial to the individual patient was never strong but neither was the evidence that the practice should be abandoned. It appears that an evidential basis for advising either for or against the provision of annual checks is overdue. This study aimed to evaluate outcomes for women attending one primary care-based IUD service in terms of whether or not they attended for frequent checks.

\section{Methods}

\section{Population}

The study used a retrospective crosssectional design and examined case records of women registered with the practice who had been fitted with an IUD or IUS between 1976 and 2005 from a GP surgery in Warwickshire with a long history of providing an IUD service. Inclusion criteria were a device in place for a minimum of 2 years and a complete set of legible records covering the time from insertion to removal or the study endpoint (March 2007). This was in order to avoid problems associated with insertion and its aftermath and also to clearly delineate those attending frequently from those who did not.

\section{Study definitions}

Each time period during which an individual IUD was in situ was treated as a separate 'IUD episode'. Definitions were drawn up to specify what constituted an IUD check, what was a reasonable time interval for a frequent check to occur, and whether a check was systematic (with evidence of an invitation) or opportunistic. 
Frequent IUD checks were categorised as having taken place when there was an average gap of less than 2 years between checks, a lack of a frequent IUD check being defined as a gap of 2 years or more. Similarly, a list of definitions was drawn up to specify when a failed attendance for a check had occurred.

Possible adverse events were divided into major (anaemia, pelvic inflammatory disease, intrauterine or ectopic pregnancy, expulsion or translocation) or minor (e.g. problematic menstrual change, insignificant discharge, patient unable to feel threads that are present, referral for lost threads with the device still in situ, etc.).

\section{Data extraction}

The records, both manual and computer-based, were individually searched, including hospital and family planning clinic letters and test results. Data were extracted by one author (IBD) for demographic details, type of IUD and date inserted, the date of removal if appropriate, the date(s) of any IUD checks, the date and type of any adverse events, and the date of any failed attendances for checks. To reduce the tendency to bias that might result from data-collection by a single individual, all events that could be attributed to a gynaecological cause were recorded, whatever their nature. A second opinion (from RJMcM) was obtained in cases of uncertainty. Data extraction was tested by piloting and dual extraction on a random selection of 20 records.

\section{Analyses}

The primary outcome of the study was the time to first major adverse event comparing frequent and infrequent IUD check attenders for systematic IUD checks (see above for definitions), considering all recorded IUD episodes. A sample size of 50 in each group, with a total number of events in the two groups of 75 , at

Table 1 Age and ethnicity of patients and details of devices used

\begin{tabular}{lr}
\hline Parameter & Quantity \\
\hline Patients ( $n)$ & 272 \\
IUD episodes ( $n)$ & 423 \\
Mean age of patients at insertion of each & $34.4(33.6-35.1)$ \\
device [years (95\% CI)] & \\
Ethnicity [ $(\%)$ ] & $253(93.0)$ \\
$\quad$ White British, White Other & $1(0.4)$ \\
Mixed & $12(4.4)$ \\
Asian, British Asian & $6(2.2)$ \\
Black, Black British & $291(68.8)$ \\
Details of devices [ $n$ (\%)] & $125(29.6)$ \\
Copper IUD & $7(1.6)$ \\
IUS (Mirena ${ }^{\circledR}$ ) & $4.19(2.95-5.43)$ \\
Plastic IUD & \\
Median duration of IUD use [years (IQR)] & \\
\hline Cl, confidence interval: IQR, interquartile range; IUD, intrauterine device; \\
IUS, intrauterine system.
\end{tabular}

$5 \%$ level two-sided log rank test for equality of survival curves will have $80 \%$ power to detect a difference of $10 \%$ event rate in frequent IUD check attenders and $30 \%$ (three-fold) event rate in infrequent IUD check attenders (a constant hazard ratio of 1.912).

Survival analyses were performed using SPSS Version 15.0 software (IBM, Portsmouth, UK), 'survival' being the statistical term used when an event has not yet occurred, ('censoring' being the term used when the event does not occur before the period of observation finishes). Time to first adverse event in frequent and infrequent IUD check attenders was compared using log rank test. Kaplan-Meier survival plots were also produced. An IUD episode was deemed to be censored if an adverse event had not occurred at the time of device removal or the end of the study period.

In addition, the primary analysis was repeated considering all checks and minor adverse events only, and any relationship between a history of defaulting from checks and the occurrence of adverse events investigated (log rank test).

\section{Results}

A total of 525 patients were identified using 828 devices, being fitted between July 1976 and February 2005. Of these, 423 IUD episodes were suitable for inclusion from a total of 272 patients (Table 1). The 405 excluded episodes were mostly unsuitable through being of too short duration (301), otherwise due to missing (81) or illegible data (7); an additional seven episodes were discarded for other reasons. Patients ranged in age from 16.3 to 55.4 years and were predominately of White ethnic origin.

The maximum number of checks performed was 13 in the case of an IUD episode that lasted 15.8 years. No checks at all occurred in 29 (6.9\%) IUD episodes. There were no defaults from checks recorded in 243 $(57.5 \%)$ IUD episodes. The maximum number of defaults was nine, seen in two IUD episodes that lasted 8.4 and 11.5 years. Minor adverse events were by far the most frequent, occurring in 364 (86.1\%) IUD episodes compared to $71(16.8 \%)$ that showed a major event (Table 2).

Table 2 The major events and the number (and percentage) of intrauterine device (IUD) episodes in which they occurred

\begin{tabular}{lc}
\hline Event & IUD episodes* $[\boldsymbol{n}(\%)]$ \\
\hline Anaemia & $48(11.35)$ \\
Pelvic inflammatory disease & $25(5.91)$ \\
Pregnancy, intrauterine & $4(0.95)$ \\
Pregnancy, ectopic & $1(0.24)$ \\
Pregnancy and expulsion combined & $1(0.24)$ \\
Expulsion & $2(0.48)$
\end{tabular}

* Some IUD episodes had more than one major event, hence there are more events recorded (81) than IUD episodes in which they occurred (71). 


\section{Relationship between checks and adverse events}

No evidence was found that regular IUD checks helped the women avoid adverse events.

\section{Systematic checks}

There was no significant difference in the survival distributions of time to first major adverse event between frequent and infrequent attenders considering systematic checks only (Figure 1, log rank test $\chi^{2}=0.037$, $\mathrm{df}=1, p=0.847$ ). Time taken for the event to happen in $25 \%$ of cases was 2331 days in frequent attenders compared to 3031 days in infrequent attenders. The survival distributions of time to first adverse event of any type were significantly different between the two groups (log rank test $\left.\chi^{2}=15.03, \mathrm{df}=1, p<0.001\right)$. Median survival times were 72 days for frequent attenders and 397 days for infrequent attenders.

\section{All checks}

The survival distributions of time to the first major adverse event, when any type of checks were considered, were significantly different between frequent and infrequent attenders $\left(\log\right.$ rank test $\chi^{2}=4.85, \mathrm{df}=1$, $p=0.028)$. This was also the case for the time to first adverse event of any type (i.e. major and minor events combined) (log rank test $\chi^{2}=8.44, \mathrm{df}=1, p=0.004$; median time to any adverse event was 217 days in frequent and 644 days in infrequent attenders).

Frequent attenders presented earlier for anaemia when any type of device check was considered (log rank test $\chi^{2}=4.68, \mathrm{df}=1, p=0.031$ ) but no significant difference was seen between frequent and nonfrequent attenders when systematic checks only were considered (log rank test $\left.\chi^{2}=0.01, \mathrm{df}=1, p=0.922\right)$.

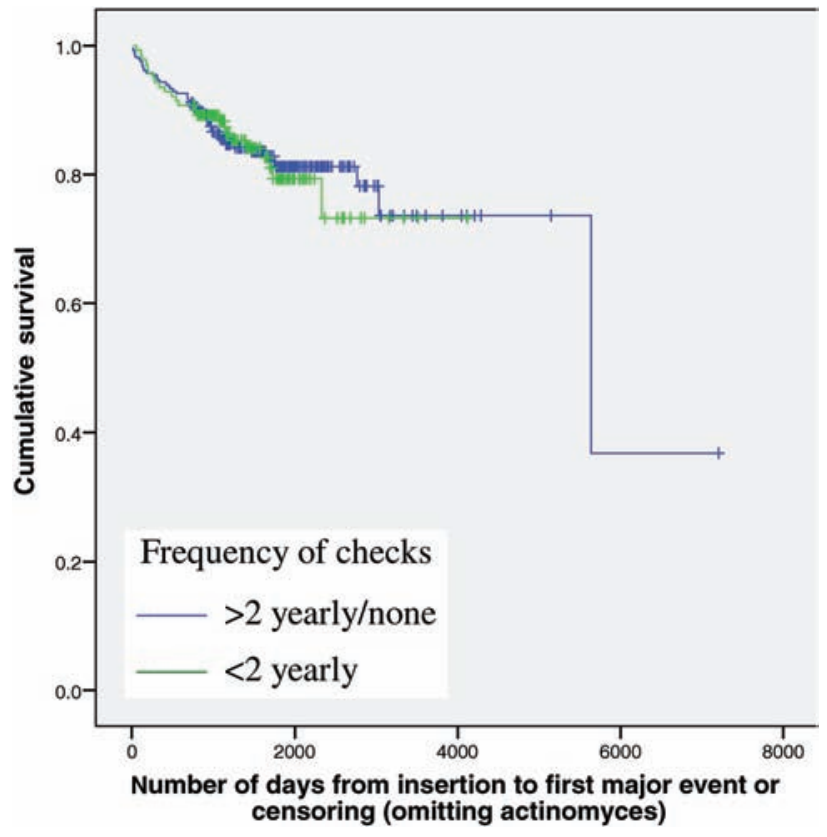

Figure 1 Number of days from insertion of intrauterine device or intrauterine system to first major event in patients receiving frequent or infrequent systematic checks.
The same relationships were found when only copper or plastic devices were investigated, but at a high level of censoring.

\section{Defaulting from IUD checks}

There was no significant difference between those individuals who did or did not default from IUD checks at least once in a 2-year period in terms of time to major adverse event [ 75 th percentile survival time was 2768 vs 3031 days (log rank test $\left.\left.\chi^{2}=0.99, p=0.319\right)\right]$. Frequent defaulters presented with any adverse event significantly later than infrequent defaulters [median 225 vs 783 days, respectively (log rank test $\left.\left.\chi^{2}=5.10, p=0.024\right)\right]$.

\section{Discussion}

\section{Statement of principal findings}

No evidence has been obtained from this study that women attending for routine IUD checks (following an invitation from a clinician) experience major adverse events later than infrequent attenders. When all checks are considered, patients having frequent checks presented earlier with problems than those that did not. Taken together this suggests that women can be left to make the decision whether to attend in response to a possible problem as opposed to undergoing regular recall.

\section{Strengths and weaknesses of the study}

This study took place in a single primary care setting with a low turnover of patients meaning that a large group of women receiving IUDs over a long period of time were available for study. However, in any study from a single centre there may be issues of generalisability. Patients were largely White, and in their early 30 s, and so caution should be taken in extrapolating the results further. It is also possible that the sample size was too small to recognise differences in the presentation of rare problems.

The counterintuitive finding that frequent check attenders presented earlier with problems than infrequent attenders may be explained as follows. Data were not extracted blind to women concerned; however, only data available in the records were utilised. A strict definition was devised to distinguish attendances for IUD-related problems from opportunistic checks. It was nonetheless possible that the two were confused; more checks would then have appeared to have occurred than was the case and they would all have been associated with adverse events.

Similarly, the presence of minor symptoms is extremely common in patients using the IUD, and it is possible that when a woman attended for a routine check, such symptoms were reported and therefore recorded in the notes when they were more an observation than a complaint of a problem. This could have biased the analysis against those having more frequent checks.

\section{Defaulting and its lack of consequences}

The overall impression is that patients were making reasonable decisions as to whether to attend or not, 
and were able to assess for themselves whether they were in need of professional attention.

\section{Comparison with other studies}

Previous research on this topic has generally been from the developing world, is not recent, and rarely looks at surveillance after the first year. ${ }^{4-6}$ An exception is Neuteboom et al. ${ }^{7}$ who compared results before and after a follow-up protocol changed in 2001 from four visits in the first year to a 6-week check then annually and found no difference in outcome. The patient group receiving the later schedule of care (which had not been running for long) inevitably is smaller, with a short duration of follow up and a larger proportion of IUSs (Mirena ${ }^{\circledR}$ ) compared to the alternative group. The authors focused on unrecognised expulsion as the main reason for a check and did not comment on problems such as slowly developing anaemia.

\section{Meaning of the study: possible implications for clinicians or policymakers}

No evidence has been found here of benefit from frequent IUD checks: patients attending or defaulting systematic checks showed no significant difference in terms of time to major event. Considering major events, it is difficult to see how a systematic annual check would help the woman to avoid most of these; they may occur predominantly soon after IUD insertion (e.g. pregnancy, pelvic inflammatory disease, expulsion) or there may be no intervention available that would prevent the problem (e.g. pregnancy). Prompt replacement of an expiring device may avoid unplanned pregnancy but this requires the carer to react to an up-to-date register, not frequent checks. It is possible, however, that the latter would help ensure that the register remained accurate, and the patient received prior warning of the impending need for replacement. Expulsion occurring some interval after insertion may be identified at a routine check, but with annual checks it is unlikely that this would be identified in time to avoid unwanted pregnancy.

A possible useful role of a routine check might be to encourage and re-educate the woman in self-checking. Anaemia might be the one major event that frequent checks might help the woman avoid if a history of increased menstruation was identified and acted upon; there is no evidence that this occurred in the present study population. It could, however, be argued that as frequent checks resulted in the anaemia being diagnosed sooner than in non-attenders, the patients benefited from earlier management of the problem.

\section{Unanswered questions and future research}

Patients will commonly be advised to attend for their first check within a few weeks of IUD insertion, and this would possibly allow early diagnosis of some of the abovementioned problems. It is to be regretted that the quality of the data available did not allow the value of a first early check to be investigated.

Further research is desirable to establish whether the final conclusions drawn here are applicable to ethnic minority patients.

\section{Conclusions}

Notwithstanding the difficulties caused by the use of retrospective data, no evidence has been found that frequent routine checks conferred any protection from adverse events for patients who appeared able to accurately judge for themselves in many cases as to whether the service would be of benefit. Caution needs to be exercised before generalising to the wider population of IUD users but this study suggests that regular annual recall of IUD users for routine checks is unnecessary.

Acknowledgements The authors are grateful to Andrea Roalfe for providing initial statistical advice.

Funding The Research Support Facility (RSF) of Primary Care Clinical Sciences at the University of Birmingham provided funding for this study from its small grant programme.

Competing interests Dr Isabel Draper and Professor Richard McManus work in general practices that benefit from fees generated by IUD checks.

Provenance and peer review Not commissioned; externally peer reviewed.

\section{References}

1 The Royal Australian and New Zealand College of Obstetricians and Gynaecologists. College Statement: Intrauterine Contraceptive Devices and Infection. 2007. http:// www.ranzcog.edu.au/publications/statements/C-gyn3.pdf [accessed 21 March 2010].

2 American Academy of Family Physicians. Intrauterine Device (IUD). 2000. http://familydoctor.org/online/famdocen/home/ women/contraceptive/319.html [accessed 21 March 2010].

3 National Institute for Health and Clinical Excellence. Longacting Reversible Contraception: The Effective and Appropriate Use of Long-acting Reversible Contraception (Clinical Guidance CG30). 2005. http://www.nice.org.uk/nicemedia/ live/10974/29912/29912.pdf [accessed 21 March 2010].

4 Bang S. Korea: the relationship between IUD retention and check-up visits. Stud Fam Plann 1971;2:110-112.

5 Hubacher D, Fortney J. Follow-up visits after IUD insertion. Are more better? J Reprod Med 1999;44:801-806.

6 Janowitz B, Hubacher D, Petrick T, et al. Should the recommended number of IUD revisits be reduced? Stud Fam Plann 1994;25:362-367.

7 Neuteboom K, de Kroon CD, Dersjant-Roorda M, et al. Followup visits after IUD-insertion: sense or nonsense? A technology assessment study to analyze the effectiveness of follow-up visits after IUD insertion. Contraception 2003;68:101-104. 